'Clínica Alemana, Valdivia, Chile. ${ }^{2}$ Hospital Base Valdivia, Valdivia, Chile. 3Instituto Pediátrico, Universidad Austral de Chile. Valdivia, Chile. ${ }^{4}$ Instituto de Farmacia, Facultad de Ciencias, Universidad Austral de Chile, Valdivia, Chile. ${ }^{a}$ Químico Farmacéutico.

Recibido el 4 de abril de 2014, aceptado el 7 de abril de 2015 .

Correspondencia a: Mónica Kyonen Eleodoro Yáñez \# 1637 , Valdivia, Chile. mkyonen@yahoo.com

\section{Comparación de dos métodos de evaluación de causalidad de sospechas de reacciones adversas a medicamentos (RAM). 2003-2009}

\author{
MÓNICA KYONEN ${ }^{2, a}$, ISABEL FOLATRE ${ }^{3}$, \\ XIMENA LAGOS ${ }^{4, a}$, SILVIA VARGAS ${ }^{1, a}$
}

Background: Assessment of causality is an essential part of pharmacological surveillance. Aim: To compare the causality assessment of suspected adverse drug reactions (ADRs) using algorithms proposed by Karch-Lasagna $(K \& L)$ and the World Health Organization (WHO). Material and Methods: All spontaneous reports of suspected ADRs in a pediatric ward of a regional hospital were included. Causality was categorized as definitive, probable, possible, conditional or unlikely. The agreement between $K \& L$ and WHO algorithms was assessed using binomial test proportions and kappa coefficients. Results: One hundred thirty reports of ADRs in 126 patients aged 2 to 11 years were analyzed. The suspected medications were antineoplastic drugs in $59 \%$ of cases and antimicrobials in $23 \%$. The most common affected system was the skin and appendages in $35 \%$. Using $K \& \mathrm{~L}$ algorithm, causality was categorized as definitive in $10 \%$ of cases, probable in $28.5 \%$, possible in $35.4 \%$, conditional in $23.1 \%$ and unlikely in 3.0\%. Using WHO algorithm, the figures were 2.3, 34.6, 59.2, 2.3 and 1.5\%, respectively. The degree of agreement between $K \& L$ and WHO algorithms was $32.3 \%(k a p p a=0.004)$. Conclusions: $K \& L$ attributed a higher level of ADR causality than WHO algorithm.

(Rev Med Chile 2015; 143: 880-886)

Key words: Algorithms; Causality; Drug related side effects and adverse reactions; Pediatrics.

$\mathrm{E}$ 1 continuo y marcado desarrollo fisiológico de la población pediátrica tiene un impacto directo sobre la farmacocinética de los medicamentos utilizados, lo que asociado a la ausencia de formulaciones farmacéuticas apropiadas, conlleva dificultades en lograr dosificaciones y concentraciones plasmáticas adecuadas.

Muchos de los medicamentos que son de uso frecuente en niños han sido comercializados con limitada o nula experiencia con respecto a la eficacia y seguridad en esta población ${ }^{1}$. Los estudios señalan que aproximadamente $50 \%$ de los medicamentos indicados a niños es en condición "off-label" y aproximadamente $70 \%$ de los niños, recibe medicamentos bajo estas condiciones ${ }^{2}$, aumentando el riesgo de ineficacia, aparición de reacciones adversas a medicamentos (RAM), o ambos ${ }^{23,24}$.

A menudo, es difícil saber cuándo una situación clínica inesperada es una RAM o es secundaria a un deterioro de la condición clínica del paciente. Se han propuesto varios métodos para diferenciarlas, entre estos se encuentran aquellos basados en el juicio clínico o en escalas algorítmicas decisionales ${ }^{3}$.

Una revisión sistemática reciente, da cuenta de 34 métodos diferentes de evaluación para RAM, que pueden ser agrupados en tres amplias cate- 
gorías: Juicio de Expertos/Introspección Global, Algoritmos y Métodos Probabilísticos (método bayesiano $)^{22}$.

El Juicio de Expertos/Introspección es una evaluación individual, basada en el conocimiento previo y la experiencia clínica y utiliza una metodología no estandarizada para evaluar causalidad. Escalas y Algoritmos, son conjuntos de respuestas a preguntas específicas con su correspondiente puntuación que permiten calcular la probable causalidad de la RAM. Resultan útiles porque proporcionan una guía de preguntas que permite obtener información veraz, disminuyendo las omisiones de datos, subjetividad e imprecisión entre evaluadores.

El método bayesiano utiliza datos específicos en un caso, para transformar la estimación de probabilidad, previa a la exposición, en una estimación de probabilidad posterior a la exposición al fármaco; combinando esta información llega a una estimación de relación causal.

Debido a problemas de reproducibilidad y validez, ningún método está universalmente aceptado. Cada método adopta diferentes categorías de causalidad y son evaluadas utilizando diferentes criterios. Entre los algoritmos más aceptados se encuentra el de Naranjo, especialmente si se trata de reacciones adversas no conocidas.

El Hospital Base de Valdivia (HBV) cuenta desde el año 1999 con farmacovigilancia activa, realizada desde el Subdepartamento de Farmacia hacia los servicios clínicos, a través de la recepción de un formulario de notificación de sospecha de RAM. Cada una de éstas es evaluada según el Algoritmo de Causalidad de Karch y Lasagna, que clasifica las sospechas de RAM en: ciertas, probables, posibles, condicionales, improbables e inclasificables. El resultado y las recomendaciones se discuten con el médico tratante y se registran en la ficha clínica del paciente.

Hasta el año 2011, las notificaciones de sospechas de RAM en nuestro medio eran de carácter espontáneo. Desde el año 2012, son obligatorias ${ }^{5}$ y los servicios clínicos envían sus sospechas de RAM al Subdepartamento de Farmacia, donde son enviadas al Centro Nacional de Farmacovigilancia del Instituto de Salud Pública, Ministerio de Salud, Santiago de Chile, para registro, categorización según el Algoritmo de la Organización Mundial de la Salud (OMS) que clasifica las sospechas de RAM en: ciertas, probables, posibles, condicionales, improbables e inclasificables.
Los reportes y resultados de causalidad de RAM son enviados al Centro Internacional de Farmacovigilancia en Uppsala, Suecia, donde se analiza la información mundial en relación a RAM, mediante el uso del algoritmo de la OMS, se comunica estos resultados y las medidas de seguridad y prevención a los países miembros de este centro, dependiente de la Organización Mundial de la Salud, del cual nuestro país es miembro oficial desde el año 1996.

El objetivo de este estudio es comparar los resultados de evaluación de causalidad de sospechas RAM en niños, empleando 2 algoritmos decisionales: Algoritmo de la OMS, usado en los centros nacional e internacional de farmacovigilancia con el Algoritmo de Causalidad Karch y Lasagna usado en el HBV.

\section{Método}

Estudio transversal de concordancia, entre 2 métodos de evaluación de causalidad de sospechas de RAM: Algoritmo de la Organización Mundial de la Salud ${ }^{17}$ y el Algoritmo de Karch y Lasagna ${ }^{18}$, en niños hospitalizados en Pediatría, Hospital Base Valdivia entre el 01 de enero de 2003 y 31 de diciembre de 2009.

Fueron incluidas en el estudio todas las notificaciones de sospechas de RAM del Subdepartamento de Pediatría. Para cada notificación de sospecha de RAM, se recogieron datos demográficos, clínicos, farmacológicos, tratamiento de las RAM, evolución y causalidad, según el Algoritmo del Sistema Español de Farmacovigilancia, de Karch y Lasagna modificado (Anexo 1).

Se utilizó la terminología del Programa de Monitorización Internacional de Reacciones Adversas a Medicamentos (WHOART) y la clasificación Química Anatómica Terapéutica (ATC) de la OMS para agrupar las RAM por sistemas y órganos, y por grupo terapéutico de los fármacos ${ }^{6}$.

La evaluación de causalidad de cada sospecha de RAM en el HBV fue realizada por tres investigadores, un médico y dos químicos farmacéuticos en conjunto; las discrepancias fueron discutidas y consensuadas.

Mediante búsqueda presencial del investigador principal en la base de datos del Centro Nacional de Farmacovigilancia del Instituto de Salud Pública, Santiago de Chile, se obtuvo los resultados de evaluación de causalidad de este centro para 
las mismas sospechas de RAM, con el algoritmo de la OMS (Anexo 2).

Ingresaron al estudio las sospechas de RAM evaluadas como ciertas, probables, posibles, condicionales e improbables, evaluadas con ambos algoritmos.

Se excluyeron las sospechas de RAM evaluadas mediante un solo algoritmo. Los evaluadores fueron ciegos a los resultados de las evaluaciones de causalidad.

Los datos se procesaron mediante el software estadístico SPSS 15.0; se utilizó la prueba estadística kappa $(k)^{7}$ para medir la fuerza de concordancia entre los algoritmos de la OMS y Karch-Lasagna.

Este estudio fue autorizado por el Comité de Ética de la Investigación del Hospital Base Valdivia, en diciembre del año 2010; fue realizado con recursos propios y no hay conflictos de interés.

No se obtuvo consentimiento informado de los pacientes o cuidadores, dado que se trabajó con datos históricos.

\section{Resultados}

En el HBV, se encontró 180 reportes espontáneos de sospechas de RAM, que corresponde a una tasa de notificación de RAM de 1 por cada 100 pacientes pediátricos atendidos en los servicios clínicos. Los reportes correspondieron a 126 pacientes, ingresando al estudio 130 reportes. Fueron excluidos del estudio 50 reportes por las siguientes causas: Notificaciones de RAM no categorizadas mediante el algoritmo OMS $(\mathrm{n}=50)$.

Se encontraron 17 pacientes con más de un episodio de RAM, ya fuese por el mismo medicamento implicado $(\mathrm{n}=8)$ o por un medicamento de diferente grupo farmacológico $(n=9)$. El 73,6\% de las notificaciones fue realizada por médicos, $9,5 \%$ por químicos farmacéuticos y $2 \%$ por enfermeras.

El grupo etario de 2-11 años presentó la mayor frecuencia de RAM $(66,1 \%)$, seguido del grupo entre 12-14 años (17\%); el sexo masculino mostró $58 \%$ de RAM (Tabla 1).

Los sistemas/órganos más frecuentemente afectados fueron piel y anexos $(34,9 \%)$, desórdenes del sistema hepático y biliar $(16,9 \%)$ y desórdenes de factores de la coagulación, plaquetas y sangrado (13,4\%) (Figura 1).

Los grupos terapéuticos más reportados como agentes sospechosos de RAM fueron los antineoplásicos $(58,9 \%)$, representados por L-asparaginasa y 6 mercaptopurina; antibióticos: cloxacilina y

Tabla 1. Distribución de sospechas de RAM por grupo etario

\begin{tabular}{|lccc|}
\hline Grupo de edad & n de reportes & Total reportes (\%) & n total pacientes atendidos* \\
\hline -27 días & 1 & 0,7 & 754 \\
\hline 2 días-23 meses & 21 & 16,1 & 6.778 \\
\hline -11 años & 86 & 66,1 & 6.471 \\
$12-14$ años & 22 & 17,0 & 1.583 \\
\hline Total & 130 & 100 & 15.586 \\
\hline
\end{tabular}

*Datos obtenidos del Subdepartamento de Información de la Salud del HBV.

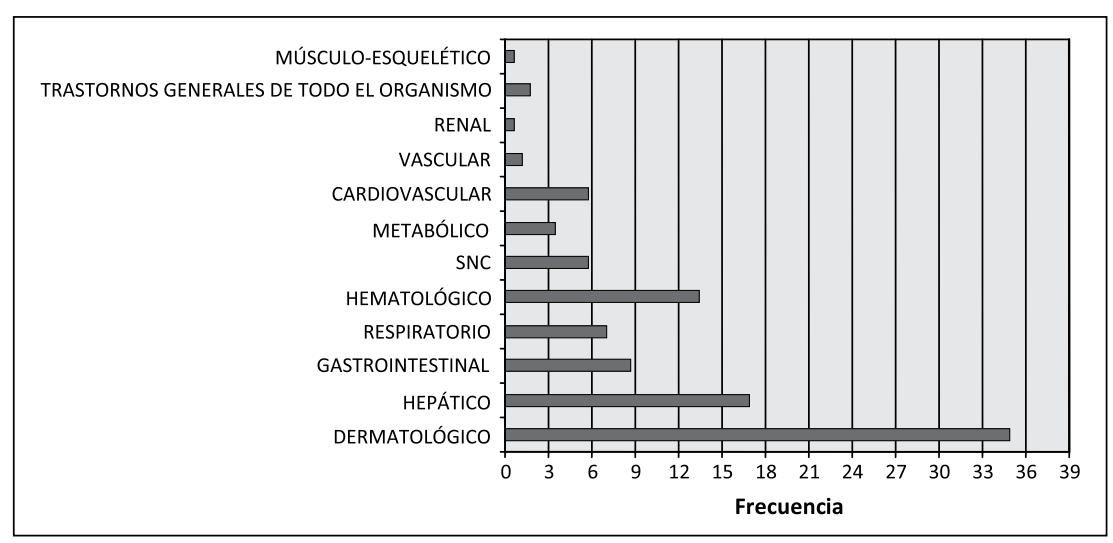

Figura 1. Distribución de RAM basado en la clasificación sistema-órgano afectado. 


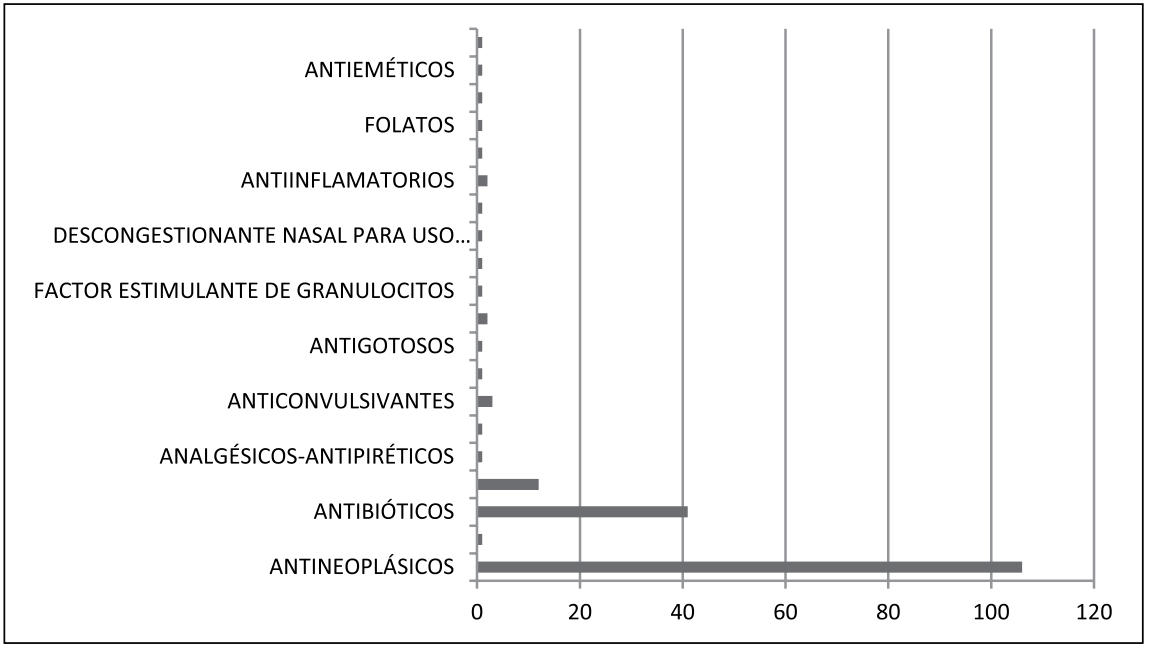

Figura 2. Distribución de las sospechas de RAM basados en la clasificación por grupo terapéutico.

Tabla 2. Evaluación de causalidad de las sospechas de RAM

\begin{tabular}{|c|c|c|c|c|c|c|c|}
\hline $\begin{array}{l}\text { Categorías de } \\
\text { causalidad }\end{array}$ & $\begin{array}{c}\text { n sospechas } \\
\text { de RAM } \\
\text { Karch \& Lasagna }\end{array}$ & $\%$ & $\begin{array}{l}\text { n sospechas } \\
\text { de RAM } \\
\text { OMS }\end{array}$ & $\%$ & $\begin{array}{c}\text { Z } \\
\text { proporciones }\end{array}$ & $\mathbf{p}$ & $\begin{array}{c}\text { K-L \& OMS } \\
\% \text { de acuerdo } \\
\text { Índice Kappa (K) }\end{array}$ \\
\hline Definitiva /Cierta & 13 & 10,0 & 3 & 2,3 & 2,584 & $\begin{array}{c}0,00 \\
(p<0,01)\end{array}$ & $\begin{array}{c}0,8 \\
K=0,000 \\
\text { IC } 95 \%(-1,020 ; 1,020)\end{array}$ \\
\hline Probable & 37 & 28,5 & 45 & 34,6 & $-1,058$ & $\begin{array}{c}0,29 \\
(p>0,05)\end{array}$ & $\begin{array}{c}9,2 \\
K=0,000 \\
\text { IC } 95 \%(-0,196 ; 0,196)\end{array}$ \\
\hline Posible & 46 & 35,4 & 77 & 59,2 & $-3,843$ & $\begin{array}{c}0,00 \\
(p<0,01)\end{array}$ & $\begin{array}{c}20,8 \\
K=0,000 \\
\text { IC } 95 \%(-0,137 ; 0,137)\end{array}$ \\
\hline $\begin{array}{l}\text { Condicional/ } \\
\text { Improbable }\end{array}$ & 34 & 26,2 & 5 & 3,8 & 5,058 & $\begin{array}{c}0,00 \\
(p<0,01)\end{array}$ & $\begin{array}{c}1,5 \\
K=0,000 \\
\text { IC } 95 \%(-0,818 ; 0,818)\end{array}$ \\
\hline Total & 130 & 100 & 130 & 100 & & & $\begin{array}{c}32,3 \text { Kappa } \\
\text { IC } 95 \%(-0,102 ; 0,109) \\
\text { K }=0,004\end{array}$ \\
\hline
\end{tabular}

ceftriaxona $(22,8 \%)$ y glucocorticoides (prednisona y dexametasona) (6,7\%) (Figura 2$)$.

El acuerdo de evaluación de causalidad entre el algoritmo de Karch y Lasagna y el algoritmo OMS fue $32,3 \%$ (Kappa 0,004) siendo para la categoría probable un acuerdo 9,2\% (Kappa 0,0) y para la categoría posible 20,8\% (Kappa 0,0) (Tabla 2).

\section{Discusión}

La baja tasa de notificaciones de sospechas de RAM $^{29,30}$ refleja un sistema de notificación aún incipiente, que puede mejorar mediante actividades de motivación y capacitación del equipo de salud. Una revisión sistemática de 102 estudios prospec- 
tivos $^{9}$ de RAM en niños, encontró gran variación cuando se compararon las tasas de incidencia reportadas desde estudios observacionales; entre los estudios, 36 estimaron una tasa de incidencia para RAM durante la hospitalización desde $0,6 \%$ a $16,8 \%$ de los pacientes; considerando que una de las principales dificultades, cuando se comparan tasas de incidencia para RAM, es que los estudios difieren en la calidad metodológica, escenario clínico, características de la población y duración del estudio.

Los resultados del análisis de fuerza de concordancia en la evaluación de los algoritmos de Karch y Lasagna y OMS son bajos; probablemente la exclusión de 50 sospechas de RAM $(27,7 \%)$ tiene un rol en el análisis estadístico.

Un estudio observacional retrospectivo ${ }^{13}$ refirió tres factores que pueden influir en la labor de un evaluador, ellos son: falta de experiencia en evaluación de la causalidad; conocimiento por los evaluadores del cuadro clínico del paciente y el conocimiento de casos similares.

Con el algoritmo de Karch y Lasagna se clasificaron las sospechas de RAM con un mayor puntaje de causalidad, que pudiera ser explicado por la ventaja de contar con las historias clínicas de los pacientes.

Un factor limitante en la evaluación de causalidad empleando ambos algoritmos, fue la información incompleta en los formularios de notificación, ya que el análisis depende de la calidad de la información obtenida.

Las categorías de causalidad, según Karch y Lasagna, observadas con mayor frecuencia fueron Probable y Posible, resultado similar al obtenido con el algoritmo OMS, tal como informa la literatura. Macedo et al. demostraron que las categorías Probable y Posible fueron las más comunes, $68 \%$, en la evaluación de causalidad por el método de introspección global ${ }^{19}$.

Estudios que emplearon el algoritmo de $\mathrm{Na}-$ ranjo $^{20}$ dan cuenta de $50 \%$ de episodios de RAM considerados como Posibles y menos de 10\% como Definitivos; sin embargo, cuando el algoritmo OMS se aplicó en la clínica, 62\% de las RAM detectadas fueron caracterizados como Definitivas y menos de $1 \%$ como Posible ${ }^{13}$. Este aumento en la categoría Definitiva se atribuyó a información más completa disponible en el momento de la evaluación.

La literatura describe que los algoritmos tendrían mayor fuerza de concordancia entre calificadores de un panel de expertos que utilizaron el Juicio Clínico ${ }^{22}$; la fuerza de concordancia de éstos varió de $41 \%$ a 57\% (kappa $=0,21-0,37$, con un coeficiente de fiabilidad $\mathrm{r}=0,49$ ); cuando los mismos individuos utilizaron el algoritmo de Naranjo la fuerza de concordancia varió entre 83\% y $92 \%$ (kappa $=0,69-0,86$, con un coeficiente de fiabilidad $r=0,92$ ), siendo este aumento estadísticamente significativo ${ }^{16}$.

Los algoritmos son procesos complejos en los que existen muchas posibilidades de falta de concordancia, diferencias en los resultados de evaluación por dos evaluadores o diferentes resultados por un mismo evaluador a lo largo del tiempo ${ }^{21}$.

\section{Conclusiones}

El algoritmo de Karch y Lasagna es un método de evaluación que hemos empleado desde el año 1999, logrando información que resulta en una categorización de las RAM de acuerdo a lo publicado en la literatura, sin embargo, este estudio mostró falta de concordancia entre los resultados de ambos métodos de evaluación, que podrían atribuirse al número de reportes excluidos, y a la calidad de la información de la RAM.

Creemos necesario aumentar las notificaciones de sospechas de RAM, mediante difusión a los usuarios, educación y formación de los equipos de salud. Continuaremos usando el algoritmo de Karch y Lasagna y, a futuro, sería de interés replicar este estudio con mayor número de reportes. 


\section{Anexo 1. Algoritmos de causalidad Karch \& Lasagna}

\begin{tabular}{|l|l|l|}
\hline Método & Criterio & Escala de Probabilidad \\
\hline $\begin{array}{l}\text { Algoritmo } \\
\text { Karch-Lasagna } \\
\text { modificado }\end{array}$ & $\begin{array}{l}\text { Secuencia Temporal: Intervalo de administración del medicamento } \\
\text { y el efecto indeseable } \\
\text { Conocimiento previo: Grado de conocimiento en la bibliografía de } \\
\text { la relación F-RA }\end{array}$ & $\begin{array}{l}\text { Valores atribuidos } \\
\text { por Karch-Lasagna } \\
\text { para una RAM } \\
\text { clasificada como: } \\
\text { Efecto de la Retirada: Evolución del efecto indeseable } \\
\text { Readministración: Efecto de la reexposición al F sospechoso }\end{array}$ \\
& Existencia de causa alternativa al medicamento $>0=8$ \\
& & $\begin{array}{l}\text { Probable } 6-7 \\
\text { Posible 4-5 } \\
\text { Condicional 1-3 } \\
\text { Improbable }<1\end{array}$ \\
\hline
\end{tabular}

\section{Anexo 2. Algoritmo de causalidad OMS}

\begin{tabular}{|l|l|l|}
\hline Método & & \\
\hline Algoritmo OMS & $\begin{array}{l}\text { Evento clínico, con una relación temporal plausible a la administración del medicamen- } \\
\text { to, no puede ser explicado por la enfermedad de base u otras drogas. La respuesta a } \\
\text { la suspensión de la droga debería ser clínicamente plausible. Debe ser definido usando } \\
\text { un procedimiento de readministración si es necesario }\end{array}$ & Cierta \\
\hline & $\begin{array}{l}\text { Evento clínico, con una secuencia temporal razonable a la administración del medica- } \\
\text { mento, e improbable que se atribuya a la enfermedad de base u otra droga, tiene una } \\
\text { respuesta razonable a la suspensión de la droga }\end{array}$ & Probable \\
\hline & $\begin{array}{l}\text { Evento clínico, con una secuencia temporal razonable a la administración del medi- } \\
\text { camento, podría ser explicado por la enfermedad de base u otro medicamento. La } \\
\text { información sobre la suspensión de la droga puede faltar o no ser clara }\end{array}$ & Posible \\
\hline & $\begin{array}{l}\text { Evento clínico, la cual es esencial tener más datos para una evaluación apropiada o los } \\
\text { datos adicionales se están examinando }\end{array}$ & Condicional \\
\hline & $\begin{array}{l}\text { Evento clínico, con una secuencia temporal a la administración del medicamento, la } \\
\text { cual hace improbable una relación causal, y las cuales otros medicamentos, o enfer- } \\
\text { medad de base proveen una explicación plausible }\end{array}$ & Improbable \\
\hline & $\begin{array}{l}\text { Evento clínico no puede ser juzgado debido a que la información es insuficiente o } \\
\text { contradictoria, y que no puede ser verificada o completada en sus datos }\end{array}$ & Inclasificable \\
\hline
\end{tabular}

\section{Referencias}

1. Aagaard L, Christensen A, Hansen EH. Information about adverse drug reactions reported in children: a qualitative review of empirical studies. Br J Clin Pharmacol 2010; 70 (4): 481-91.

2. Fernández-Llamazares C, Manrique S, Sanjurjo S. Seguridad en el uso de medicamentos en pediatría. Arch Argent Pediatr 2011; 109 (6): 510-8.

3. Altavilla A, Manfredi C, Baiardi P, Dehlinger-Kremer M, Galletti P, Alemany Pozuelo A, et al. Impact of the new European paediatric regulatory framework on ethics committees: overview and perspectives. Acta Pediatr 2012; 101 (1): e27-e32.
4. Arimone Y, Miremont-Salamé G, Haramburu F, Molimard M, Moore N, Fourrier-Réglat A, et al. Inter-expert agreement of seven criteria in causality assessment of adverse drug reactions. Br J Clin Pharmacol 2007; 64 (4): 482-8.

5. DS 3/2010 "Reglamento del Sistema Nacional de Control de los Productos farmacéuticos de uso humano" Ministerio de Salud; Subsecretaría de Salud Pública, diciembre de 2011.

6. WHO Collaborating Centre for Drug Statistics Methodology. ATC classification index with DDDs, Oslo 2009: WHO Collaborating Centre for Drug Statistics Methodology (consultado el 1 de agosto de 2013).

7. Landis JR, Koch GG. The measurement of observer 
agreement for categorical data. Biometrics 1977; 33: 159-74.

8. Alonso P, Otero MJ, Maderuelo JA. Ingresos hospitalarios causados por medicamentos: incidencia, características y coste. Farmacia Hosp 2002; 26 (2): 77-89.

9. Smyth R, Mary D, Gargon E, Kirkham J, Cresswell L, Golder S, et al. Adverse Drug Reation in Children. A systematic review. PLos One 2012; 7 (3): 1-22.

10. Kyonen M, Folatre I, Zolezzi P, Badilla V, Marín F. Reacciones Adversas a L-asparaginasa en pacientes con leucemia Linfoblástica Aguda. Rev Med Chile 2006; 134: 1530-4.

11. Arimone Y, Begaud B, Miremont-Salame G, Fourrier-Reglat A, Molimard M, Moore N, et al. A new method for assessing drug causation provided agreement with experts' judgment. J Clin Epidemiol 2006; 59: 308-14.

12. Ortiz MI, Roldán Rivera S, Escamilla Acosta MA. Side Effects of Vincritine and L-asparaginasa in Patients with acute Lymphoblastic Leukemia in a Mexican Pediatric Hospital. Pharmacology\& Pharmacy 2013; 4: 347-54.

13. Lei HS, Ab Rahman AF, Haq AHS. Adverse Drug Reation Reports in Malasya: Comparison of Causality Assesments Malasyan Journal of Pharmaceutical Sciences 2007; 5 (1): 7-17.

14. Dormann H, Muth-Selbach U, Krebs S, Criegee-Rieck $\mathrm{M}$, Tegeder I, Schneider HT, et al. Incidence and costs of adverse drug reactions during hospitalization-computerised monitoring versus stimulated spontaneous reporting, Drug Safety 2000; 22: 161-8.

15. Classen DC, Pestotnik SL, Evans S, Burke JP. Computerized surveillance if adverse drug events in hospital patients, Journal of American Medical Association 1991; 266: 2847-51.

16. Doherty MJ. Algorithms for assessing the probability of an Adverse Drug Reaction. Respiratory Medicine CME 2 2009; 64: 63-7.

17. Anónimo.Vigilancia de la seguridad de los medicamentos.Guía para la instalación y puesta en funcionamiento de un centro de Farmacovigilancia. Uppsala Monitoring Centre, 2001.

18. Karch F, Lasagna L. Toward the operational identification of adverse drug reactions. Clin Pharmacol Ther 1977; 21: 247-54.

19. Macedo AF, Marque FB, Ribeiro CE, Teixeira F. Causality assessment of adverse drug reactions: Comparison of the results obtained from published decisional algorithms and from the evaluations of an expert panel, according to different levels of imputability, Journal of Clinical Pharmacy and Therapeutics 2003; 28: 137-43.
20. Naranjo C, Busto U, Sellers EM, Sandor P, Ruiz I, Roberts EA, et al. A method for estimating the probability of adverse drug reactions. Clin Pharm Ther 1981; 30: 239-45.

21. Agbabiaka TB, Savovic J and Ernst E. Methods for Causality Assesment of Adverse Drug Reactions Drug Safety. A Systematic Review 2008; 31(1): 21-37.

22. Théophile H, Arimone Y, Salamé-Miremova G, Moore $\mathrm{N}$, et al. Comparison of three methods (consensual expert judgement algorithmic and probabilistic approachs) of causality assessment of adverse drug reactions. Drug safety 2010; 33 (11): 1045-54.

23. Star K, Norén N G, Nordin K, Edwards IR. Suspected adverse drug reactions reported for children worldwide. Drug Safety 2011; 34 (5): 415-28.

24. European Medicines Agency. ICH topic E 11. Clinical investigation of medicinal products in the paediatric population. 2001 Jan. CPMP/ICH/2711/99 [online]. Available from URL: http://www.ema.europa.eu/pdfs/ human/ich/271199en.pdf [consultada el 10 de Junio 2009].

25. Aldea A, García Sánchez-Colomer E, Fernández Quintana M, García Sáiz M. Paediatric adverse drug reactions reported to the Spanish Pharmacovigilance System from 2004 to 2009. Eur J Clin Pharmacol 2012; 68 (9): 132938.

26. Capellà D, Laporte JR, Tognoni G. notificación espontánea de reacciones adversas. En: Principios de epidemiología del medicamento. Barcelona, España: $2^{\mathrm{a}}$ ed. Masson-Salvat Medicina 1993; (8): 147-69.

27. Hazell L, Shakir S. Under-Reporting of Adverse Drug Reactions: A Systematic Review. Drug Safety 2006; 29 (5): 385-96.

28. Clarkson IA, Provasi D, Pandolfini CH, Bonati M. Incidence of adverse drug reactions in paediatric in/ out-patients: a systematic review and meta-analysis of prospective studies. Clin Pharmacol 2001; 52: 77-83.

29. Le J, Nguyen T, Law A, Holding J. Adverse Drug Reactions among Children over a 10-Year Period. Pediatrics 2006; 118: 555-62.

30. Napoleone E. Children and ADRs (Adverse Drug Reactions). Italian Journal of Pediatrics 2010; 36: 4.

31. Speranza N, Lucas L, Telechea H, Santurio A, Giachetto G, Nanni L. Reacciones adversas a medicamentos en niños hospitalizados: un problema de salud pública. Rev Med Urug 2008; (24) 3: 161-6.

32. Miremont G, Haramburu F, Bégaud B, Péré JC, Dangoumau J. Adverse drug reactions: physicians' opinions versus a causality assessment method. Eur J Clin Pharmacol 1994; 46 (4): 285-9. 